\author{
Hui-Min Hsieh ( $\square$ hsiehhm@gmail.com ) \\ Kaohsiung Medical University \\ Cheng-Ting Shen \\ Kaohsiung Municipal Ta-Tung Hospital \\ Fang-Ming Chen \\ Kaohsiung Municipal Ta-Tung Hospital
}

8 Research Square

Preprints are preliminary reports that have not undergone peer review.

They should not be considered conclusive, used to inform clinical practice, or referenced by the media as validated information.

\section{Moderation Effect of Mammography Screening Among Women with Multiple Chronic Conditions}

\author{
Research Article \\ Keywords: Breast cancer screening, comorbidity, mammography participation, health policy, women health. \\ Posted Date: October 18th, 2021 \\ DOI: https://doi.org/10.21203/rs.3.rs-962596/v1 \\ License: (c) (i) This work is licensed under a Creative Commons Attribution 4.0 International License. Read Full License
}

Version of Record: A version of this preprint was published at Scientific Reports on February 10th, 2022. See the published version at https://doi.org/10.1038/s41598-022-06187-7. 


\section{Abstract}

Purpose: To investigate the moderation effects of mammography screening on early stage breast cancer and all-cause mortality among women aged 50-69 years with chronic conditions in Taiwan.

Method: We used a matched cohort design with four nationwide population databases, and an exact matching approach to match groups with different chronic conditions. Women population aged 50-69 years in 2010 in Taiwan were studied. A generic Charlson comorbidity index $(\mathrm{CCl})$ measure was used to identify chronic illness burden. The sample sizes of each paired matched group with $\mathrm{CCl}$ scores of $0,1,2$, or $3+$ were 170,979 using a 1-to-1 exact matching. Conditional logistic regressions with interaction terms were used to test moderation effect, and adjusted predicted probabilities and marginal effects to quantify average and incremental chronic conditions associated with outcome measures. Statistical analyses were conducted in 2020-2021.

Results: Women with more chronic conditions were less likely to participate in mammography screening or to receive early breast cancer diagnoses, but were at greater risk of mortality. However, mammography participation increased the likelihood of early breast cancer diagnosis $(\mathrm{OR}=1.48,95 \% \mathrm{Cl}=1.36-1.60)$ and decreased risk of all-cause mortality $(\mathrm{OR}=0.63,95 \% \mathrm{Cl}=0.60-0.66)$. The interaction terms of $\mathrm{CCl}$ and mammography participation indicated significantly increased benefits of early breast cancer diagnosis and decreased risk of all-cause mortality as chronic illness increased.

Conclusions: Mammography participation significantly moderated the link between comorbidity and outcome measures among women with chronic conditions. Hence, it is important for public health policy to promote mammography participation for women with multiple chronic conditions.

\section{Key Message}

1. Comorbidity substantially affects breast cancer risk and cancer stage at diagnosis. However, most existing studies suggest that women with chronic conditions are less likely to participate in breast cancer screening.

2. This study found mammography participation significantly moderated the link between comorbidity and outcome measures among women with chronic conditions.

3. Hence, it is important for public health policy to promote mammography participation for women with multiple chronic conditions.

\section{Introduction}

Female breast cancer is the leading cause of global cancer incidence, with an estimated 2.3 million new cases, and the fifth leading cause of cancer mortality worldwide. ${ }^{1}$ In 2020, the global cancer project (GLOBOCAN 2020) estimated increasing age-specific standardized incidence of 47.8 per million and mortality rates of 13.6 per million. ${ }^{1}$ In Taiwan, female breast cancer is also the leading cause of cancer. Age-standardized incidence rates increased from 28.4 per million in 1995 to 78.9 per million in 2018, and age-standardized mortality rates from 9.7 per million to 12.5 per million. ${ }^{2}$ To improve early diagnosis and survival outcomes, mammography has been suggested as an effective screening tool. The American Cancer Society recommends that average-risk women aged 45-54 years undergo mammography annually, and women aged 55 years or older biannually. A report from the 2013 National Health Interview Survey of America found that $69.1 \%$ of women aged 50 years or older are adherent to breast cancer screening guidelines every 2 years, while but mammography screening rates remain much lower in Asian countries. ${ }^{3}$

Cancer and chronic conditions may share common risk factors, including demographics (age, sex, ethnicity), and genetic and lifestylerelated factors (obesity, diet, physical activity, tobacco or alcohol consumption). ${ }^{4-6}$ Studies have shown that comorbidity substantially affects breast cancer risk and cancer stage at diagnosis. ${ }^{4,7-9}$ Therefore, chronic conditions play an important role in adherence to breast cancer screening guidelines for prevention and control. However, most existing studies suggest that women with chronic conditions are less likely to participate in breast cancer screening. ${ }^{10,11}$ Several plausible explanations address the negative association between comorbidity and mammography. For example, women with comorbid conditions may be deterred by physical barriers (pain, limited mobility), or by competing demands and health priorities given limited resources (time, money, energy). 7,9,10

Furthermore, unclear potential benefits of mammography among women with different health statuses, especially those aged 50-69 years, may affect health providers' referral decisions or individuals' willingness to undergo mammography. ${ }^{12-15}$ Most existing studies focus on benefits of mammography screening among older women and were conducted in the United States or Europe. ${ }^{16,17}$ For example, a systematic review by Braithwaite et al. (2016) considered seven studies regarding the benefit or harm of mammography screening in 
women aged 65 years or older in relation to comorbidity, all conducted in the United States. Their findings suggested that these women may have longer life expectancy due to screening. ${ }^{15}$ Another recent study by Demb et al. (2020) examined the effects of continuous mammography on risk of incident breast cancer and mortality among 222,088 screened women ages 66-94 years in the United States, and found higher risk of other cause of death among women with comorbidity than among those without, but similar risk of incident breast cancer in women with different comorbidity levels. Their findings suggest that older women with increasing comorbidity may have diminished benefit from continuous screening. ${ }^{12}$ Very few studies younger women with chronic conditions. There was a recent study by Beau et al. (2020) investigated the effect of chronic diseases on risk of breast cancer mortality among women aged 50-69 years in screened and non-screened regions in Denmark, and found marginal effect of mammography on breast cancer mortality in women with chronic diseases. ${ }^{18}$

Given that few studies examine the potential benefit of mammography screening among women aged 50-69 years with chronic conditions in Asian countries, we sought to use a population-based matched cohort study design to examine the potential benefit and moderation effect of mammography screening among women aged 50-69 years at various health statuses in Taiwan. Breast cancer screening policy in Taiwan is a key national cancer prevention policy. Since 2004, after passage of the National Cancer Prevention Law of 2003, Taiwan's Ministry of Health Promotion Administration initiated an organized breast cancer screening strategy covering free biannual mammography services for the entire population of women aged 50-69 years. ${ }^{19}$ The current study identified entire female population aged 50-69 yeas in 2010 and used an exact matching approach to match women at different chronic illness levels. A generic comorbidity measure was used to characterize total chronic illness burden. We then compared the effect of mammographic screening on early diagnosis of breast cancer and all-cause mortality among matched cohorts at various health statuses.

\section{Methods}

\section{Study Design and Data Source}

We used a matched cohort study design with at least 4-year follow-up using four nationwide population databases in Taiwan. The first was the National Health Insurance (NHI) administrative claims database, which includes more than $99 \%$ of Taiwan's 23 million enrollees. ${ }^{20}$ The $^{2}$ $\mathrm{NHI}$ database provides information including enrollment status, comorbid conditions, preventive care use, and primary health providers for chronic conditions. The second database was a national breast cancer screening registry, 2004-2014, which collected accurate mammography dates for participants. The third was a national cancer registry, from which accurate diagnoses of overall cancer and breast cancer could be derived, 1979-2014. ${ }^{21,22}$ The fourth was a national death registry, 2004-2014, which provides accurate death dates. We linked and analyzed these four population-based datasets with encrypted identifiers for the study population during 2020-2021 in the Health and Welfare Data Science Center of the Ministry of Health and Welfare, a government-operated national data warehouse.

\section{Ethical aspects}

The study followed the ethical standards of the Institutional Review Board of the Kaohsiung Medical University Hospital (IRB number: KMUHIRB-E(I)-20190177) and the Helsinki Declaration of the World Medical Association. Consent to Participate: Given this research was retrospective study using secondary health administrative database, patients' informed consent was waived.

\section{Study Population}

We first identified all women aged 50-69 years in $2010(n=2,564,252)$ eligible for free mammography screening in the national breast cancer screening program using the national NHI enrollment data. The study index date was defined as January 1, 2010, and the study end date as December 31, 2014. Each study cohort was followed from the index date to the first of study end date, or death. To avoid potential problems due to existing cancer disease or erroneous records, we excluded women with any record of cancer diagnosis $(n=143,619)$ or death $(n=487)$ before the index date. We then excluded women with any record of breast cancer screening services before the index date $(n=510,808)$ to ensure that all study subjects were new to mammography. To reduce potential bias due to missing data when measuring an individual's health status, we further excluded women without medical records during follow-up $(n=33,536)$. A total of $1,867,802$ women aged 50-69 years were included in this study.

To identify levels of overall chronic illness severity in the study sample, we used the Deyo-Charlson Comorbidity Index (CCl), weighting comorbid conditions from the index date to the date of mammography, incident breast cancer, death, or study end date, whichever came first. ${ }^{23,24}$ This index is an ICD-9-CM coding adaption and has been widely used by health researchers to measure general disease severity and case mix in health administrative claims databases, with low scores representing lowest risk. ${ }^{23,24}$ The study sample was classified into four groups with $\mathrm{CCl}$ scores of $0,1,2$, or $3+$. As older age is concurrent with increased risk of chronic conditions and mortality, leading to 
selection bias and incomparable samples, we used a 1-to-1 exact matching approach to match groups with different overall chronic illness severity levels based on index age in years and baseline income status ( $<$ new Taiwanese dollar [NTD] 20,000, dependent, NTD 20,00040,000 , and NTD 40,001+) to create four pairs of exactly balanced groups. ${ }^{25}$ The sample sizes of each paired matched group with CCI scores of $0,1,2$, or $3+$ were 170,979. Figure 1 shows study inclusion and exclusion criteria.

\section{Measurements and Variable Definitions Outcome of Interest}

We aimed to examine the effect of mammography on early breast cancer diagnosis and all-cause mortality among women with various comorbid conditions. The first diagnosis in the Taiwan cancer registry after the index date with ICD-9-CM diagnosis code 174 or ICD-10-CM code C50 was identified as incident breast cancer. Based on the Tumor-Nodes-Metastasis staging system of the American Joint Committee on Cancer version 7 in the National Cancer Registry, incident breast cancer stages were categorized as 0-IV and early stage (0II) or advanced stage (III, IV). We also linked data with the National Death Registry and defined all-cause mortality as any death record after the index date. In addition, we included a set of binary variables for mammography participation, and mammography access through inreach in a hospital or out-reach in a community.

\section{Other Confounding Baseline Covariates}

In addition to covariates such as the $\mathrm{CCl}$ and income status categories, we included two variables to identify health behavior characteristics (participation in any population-based pap smear cervical cancer screening program or adult physical examination program within the follow-up period). In Taiwan, the Health Promotion Administration, Ministry of Health and Welfare, provides government-initiated national population-based health promotion programs, including the two mentioned. ${ }^{26}$ Women aged older than 30 years are eligible to participate in free annual pap smear screening under the national population-based cervical cancer screening program. Adults aged 40-65 years are eligible to participate in free physical examinations to receive routine blood tests and basic physical examination every 3 years, and those older than 65 years every year. ${ }^{26}$ To address the issue of patients with multiple outpatient visits to different health care providers, we used a plurality provider algorithm to assign the most frequently visited hospitals or clinics based on administrative billing for the greatest numbers of care visits during follow-up. ${ }^{27}$ Health care institution characteristics included accreditation level (medical center, regional hospital, local hospital, clinic), certification status for mammography screening (yes/no), and geographic location (Taipei, northern, central, southern, Kao-Ping, and eastern regions).

\section{Statistical Analysis}

Descriptive analyses using chi-square tests for categorical variables and t-tests for continuous variables. The Cochran-Armitage test for trend was used to test linear trends in frequencies of outcome measures among different chronic condition levels. ${ }^{28}$ Individual-level conditional logistic regression models were used to compare outcome measures among exactly matched women with different chronic conditions. Conditional logistic regression was a proper method for matched data to address the sparse data problem and provide robust results. ${ }^{29}$ Interaction terms for mammography and $\mathrm{CCl}$ were generalized to test the moderation effects of mammography on early breast cancer diagnosis and mortality. ${ }^{30}$ If an effect exists, the moderating variable may change the direction or magnitude of the relationship between $\mathrm{CCl}$ and outcome measures and the interaction term will be statistically significant. ${ }^{30}$ In addition, to contextualize the magnitude of the mammography moderation effect, we generated adjusted predicted probabilities (APPs) and marginal effects (MEs) of the probabilities. ${ }^{31}$ These are used to quantify the average and incremental level of chronic illness associated with outcome measures. ${ }^{31,32}$ Specifically, we generated APPs and MEs of each chronic illness severity level on early breast cancer diagnosis and mortality among women who did or did not undergo mammography. For each chronic illness level, we also generated MEs to compare the likelihood of severe chronic illness on outcome measures among women who did or did not undergo mammography. Data analysis was generated using SAS $\circledast$ software, version 9.4 of the SAS System for Windows. Copyright@2020. SAS Institute Inc. product or service names are registered trademarks or trademarks of SAS Institute Inc., Cary, NC, USA. A p value $<0.05$ was considered statistically significant.

\section{Results}

Table 1 summaries baseline demographic, health behavior, and health care institution characteristics among women aged 50-69 years at different chronic illness levels in 2010. Before matching, cohorts with higher CCls were older. Mean age among women with $\mathrm{CCl} 0,1,2,3+$ in 2010 was 56.25, 57.87, 58.87, and 60.38 years, respectively. After exact matching based on index age and income status, demographic characteristics among women at different $\mathrm{CCl}$ levels were comparable. 
Table1. Study cohort demographic and clinical characteristics and primary health care providers' organizational characteristics among study women with different levels of chronic illness 
Pre-Matching Cohort

Matched Cohort ${ }^{\mathrm{a}}$

\begin{tabular}{|c|c|c|c|c|c|c|c|c|c|c|}
\hline Variables & $\mathrm{CCl}=0$ & $\mathrm{CCl}=1$ & $\mathrm{CCl}=2$ & $\mathrm{CCl}=3+$ & $\begin{array}{c}\mathrm{p}^{-} \\
\text {value }^{\mathrm{b}}\end{array}$ & $\mathrm{CCl}=0$ & $\mathrm{CCl}=1$ & $\mathrm{CCl}=2$ & $\mathrm{CCl}=3+$ & $\begin{array}{c}\mathrm{p}^{-} \\
\text {value }^{\mathrm{b}}\end{array}$ \\
\hline $\mathrm{N}$ & $1,053,018$ & 424,072 & 184,014 & 206,698 & & 170,979 & 170,979 & 170,979 & 170,979 & \\
\hline \multicolumn{11}{|l|}{$\begin{array}{l}\text { Women' } \\
\text { demographic } \\
\text { characteristics }\end{array}$} \\
\hline $\begin{array}{l}\text { Age in years } \\
(\text { Mean } \pm S T D)^{a}\end{array}$ & $\begin{array}{l}56.25 \\
( \pm 5.14)\end{array}$ & $\begin{array}{l}57.87 \\
( \pm 5.53)\end{array}$ & $\begin{array}{l}58.87 \\
( \pm 5.68)\end{array}$ & $\begin{array}{l}60.38 \\
( \pm 5.70)\end{array}$ & $<0.001$ & $\begin{array}{l}59.36 \\
( \pm 5.56)\end{array}$ & $\begin{array}{l}59.36 \\
( \pm 5.56)\end{array}$ & $\begin{array}{l}59.36 \\
( \pm 5.56)\end{array}$ & $\begin{array}{l}59.36 \\
( \pm 5.56)\end{array}$ & 1.000 \\
\hline \multicolumn{11}{|l|}{$\begin{array}{l}\text { Age categories } \\
(\mathrm{N}, \%)\end{array}$} \\
\hline $50-54$ & $\begin{array}{l}476,831 \\
(45.28 \%)\end{array}$ & $\begin{array}{l}142,370 \\
(33.57 \%)\end{array}$ & $\begin{array}{l}50,495 \\
(27.44 \%)\end{array}$ & $\begin{array}{l}40,133 \\
(19.42 \%)\end{array}$ & $<0.001$ & $\begin{array}{l}40,133 \\
(23.47 \%)\end{array}$ & $\begin{array}{l}40,133 \\
(23.47 \%)\end{array}$ & $\begin{array}{l}40,133 \\
(23.47 \%)\end{array}$ & $\begin{array}{l}40,133 \\
(23.47 \%)\end{array}$ & 1.000 \\
\hline $55-59$ & $\begin{array}{l}310,812 \\
(29.52 \%)\end{array}$ & $\begin{array}{l}125,694 \\
(29.64 \%)\end{array}$ & $\begin{array}{l}52,010 \\
(28.26 \%)\end{array}$ & $\begin{array}{l}52,004 \\
(25.16 \%)\end{array}$ & & $\begin{array}{l}49,522 \\
(28.96 \%)\end{array}$ & $\begin{array}{l}49,522 \\
(28.96 \%)\end{array}$ & $\begin{array}{l}49,522 \\
(28.96 \%)\end{array}$ & $\begin{array}{l}49,522 \\
(28.96 \%)\end{array}$ & \\
\hline $60-64$ & $\begin{array}{l}168,161 \\
(15.97 \%)\end{array}$ & $\begin{array}{l}89,201 \\
(21.03 \%)\end{array}$ & $\begin{array}{l}43,237 \\
(23.50 \%)\end{array}$ & $\begin{array}{l}53,414 \\
(25.84 \%)\end{array}$ & & $\begin{array}{l}43,052 \\
(25.18 \%)\end{array}$ & $\begin{array}{l}43,052 \\
(25.18 \%)\end{array}$ & $\begin{array}{l}43,052 \\
(25.18 \%)\end{array}$ & $\begin{array}{l}43,052 \\
(25.18 \%)\end{array}$ & \\
\hline $65-69$ & $\begin{array}{l}97,214 \\
(9.23 \%)\end{array}$ & $\begin{array}{l}66,807 \\
(15.75 \%)\end{array}$ & $\begin{array}{l}38,272 \\
(20.80 \%)\end{array}$ & $\begin{array}{l}61,147 \\
(29.58 \%)\end{array}$ & & $\begin{array}{l}38,272 \\
(22.38 \%)\end{array}$ & $\begin{array}{l}38,272 \\
(22.38 \%)\end{array}$ & $\begin{array}{l}38,272 \\
(22.38 \%)\end{array}$ & $\begin{array}{l}38,272 \\
(22.38 \%)\end{array}$ & \\
\hline \multicolumn{11}{|l|}{$\begin{array}{l}\text { Income status } \\
(\mathrm{N}, \%)^{\mathrm{a}, \mathrm{c}}\end{array}$} \\
\hline$<$ NTD 20,000 & $\begin{array}{l}150,843 \\
(14.32 \%)\end{array}$ & $\begin{array}{l}58,005 \\
(13.68 \%)\end{array}$ & $\begin{array}{l}26,069 \\
(14.17 \%)\end{array}$ & $\begin{array}{l}31,646 \\
(15.31 \%)\end{array}$ & $<0.001$ & $\begin{array}{l}25,205 \\
(14.74 \%)\end{array}$ & $\begin{array}{l}25,205 \\
(14.74 \%)\end{array}$ & $\begin{array}{l}25,205 \\
(14.74 \%)\end{array}$ & $\begin{array}{l}25,205 \\
(14.74 \%)\end{array}$ & 1.000 \\
\hline Dependent & $\begin{array}{l}280,440 \\
(26.63 \%)\end{array}$ & $\begin{array}{l}132,443 \\
(31.23 \%)\end{array}$ & $\begin{array}{l}63,314 \\
(34.41 \%)\end{array}$ & $\begin{array}{l}82,820 \\
(40.07 \%)\end{array}$ & & $\begin{array}{l}62,576 \\
(36.60 \%)\end{array}$ & $\begin{array}{l}62,576 \\
(36.60 \%)\end{array}$ & $\begin{array}{l}62,576 \\
(36.60 \%)\end{array}$ & $\begin{array}{l}62,576 \\
(36.60 \%)\end{array}$ & \\
\hline $\begin{array}{l}\text { NTD 20,000- } \\
40,000\end{array}$ & $\begin{array}{l}468,303 \\
(44.47 \%)\end{array}$ & $\begin{array}{l}180,911 \\
(42.66 \%)\end{array}$ & $\begin{array}{l}74,892 \\
(40.70 \%)\end{array}$ & $\begin{array}{l}76,528 \\
(37.02 \%)\end{array}$ & & $\begin{array}{l}67,897 \\
(39.71 \%)\end{array}$ & $\begin{array}{l}67,897 \\
(39.71 \%)\end{array}$ & $\begin{array}{l}67,897 \\
(39.71 \%)\end{array}$ & $\begin{array}{l}67,897 \\
(39.71 \%)\end{array}$ & \\
\hline NTD 40,001+ & $\begin{array}{l}153,432 \\
(14.57 \%)\end{array}$ & $\begin{array}{l}52,713 \\
(12.43 \%)\end{array}$ & $\begin{array}{l}19,739 \\
(10.73 \%)\end{array}$ & $\begin{array}{l}15,704 \\
(7.60 \%)\end{array}$ & & $\begin{array}{l}15,301 \\
(8.95 \%)\end{array}$ & $\begin{array}{l}15,301 \\
(8.95 \%)\end{array}$ & $\begin{array}{l}15,301 \\
(8.95 \%)\end{array}$ & $\begin{array}{l}15,301 \\
(8.95 \%)\end{array}$ & \\
\hline \multicolumn{11}{|l|}{$\begin{array}{l}\text { Women' health } \\
\text { behavioral } \\
\text { characteristics } \\
(\mathrm{N}, \%)\end{array}$} \\
\hline \multicolumn{11}{|l|}{$\begin{array}{l}\text { Receiving } \\
\text { population- } \\
\text { based pap } \\
\text { smear } \\
\text { screening } \\
\text { within follow } \\
\text { up period (N, } \\
\%)\end{array}$} \\
\hline No & $\begin{array}{l}448,375 \\
(42.58 \%)\end{array}$ & $\begin{array}{l}177,792 \\
(41.92 \%)\end{array}$ & $\begin{array}{l}79,555 \\
(43.23 \%)\end{array}$ & $\begin{array}{l}108,514 \\
(52.50 \%)\end{array}$ & $<0.001$ & $\begin{array}{l}78,619 \\
(45.98 \%)\end{array}$ & $\begin{array}{l}75,486 \\
(44.15 \%)\end{array}$ & $\begin{array}{l}75,122 \\
(43.94 \%)\end{array}$ & $\begin{array}{l}87,535 \\
(51.20 \%)\end{array}$ & $<0.001$ \\
\hline Yes & $\begin{array}{l}604,643 \\
(57.42 \%)\end{array}$ & $\begin{array}{l}246,280 \\
(58.08 \%)\end{array}$ & $\begin{array}{l}104,459 \\
(56.77 \%)\end{array}$ & $\begin{array}{l}98,184 \\
(47.50 \%)\end{array}$ & & $\begin{array}{l}92,360 \\
(54.02 \%)\end{array}$ & $\begin{array}{l}95,493 \\
(55.85 \%)\end{array}$ & $\begin{array}{l}95,857 \\
(56.06 \%)\end{array}$ & $\begin{array}{l}83,444 \\
(48.80 \%)\end{array}$ & \\
\hline
\end{tabular}

Note: $\mathrm{CCl}=$ modified Charlson comorbidity index, $\mathrm{NHI}=$ National Health Insurance Administration, NTD=New Taiwanese Dollar.

a Exact matching approach was used to 1-to-1 match groups of samples with different level of overall chronic illness severity based on their index age in years and baseline income status.

${ }^{b}$ P-value for comparison of patients' and health care providers' characteristics between screened and non-screened women, chi-square tests were used for categorical variables and t-tests for continuous variables.

${ }^{c}$ Income status was presented in New Taiwanese Dollar (NTD). The exchange rate between NTD and US Dollar is about 30:1 in this study. 
Receiving

population-

based adult

physical

examinations

within follow

up period (N,

$\%)$

\begin{tabular}{|c|c|c|c|c|c|c|c|c|c|c|}
\hline No & $\begin{array}{l}503,333 \\
(47.80 \%)\end{array}$ & $\begin{array}{l}166,320 \\
(39.22 \%)\end{array}$ & $\begin{array}{l}69,744 \\
(37.90 \%)\end{array}$ & $\begin{array}{l}88,006 \\
(42.58 \%)\end{array}$ & $<0.001$ & $\begin{array}{l}78,177 \\
(45.72 \%)\end{array}$ & $\begin{array}{l}66,243 \\
(38.74 \%)\end{array}$ & $\begin{array}{l}64,350 \\
(37.64 \%)\end{array}$ & $\begin{array}{l}73,608 \\
(43.05 \%)\end{array}$ & $<0.001$ \\
\hline Yes & $\begin{array}{l}549,685 \\
(52.20 \%)\end{array}$ & $\begin{array}{l}257,752 \\
(60.78 \%)\end{array}$ & $\begin{array}{l}114,270 \\
(62.10 \%)\end{array}$ & $\begin{array}{l}118,692 \\
(57.42 \%)\end{array}$ & & $\begin{array}{l}92,802 \\
(54.28 \%)\end{array}$ & $\begin{array}{l}104,736 \\
(61.26 \%)\end{array}$ & $\begin{array}{l}106,629 \\
(62.36 \%)\end{array}$ & $\begin{array}{l}97,371 \\
(56.95 \%)\end{array}$ & \\
\hline
\end{tabular}

Primary Health

Care Providers'

Characteristics

\section{Accreditation}

level $(\mathrm{N}, \%)$

\begin{tabular}{|c|c|c|c|c|c|c|c|c|c|c|}
\hline Medical Center & $\begin{array}{l}105,313 \\
(10.00 \%)\end{array}$ & $\begin{array}{l}60,118 \\
(14.18 \%)\end{array}$ & $\begin{array}{l}30,565 \\
(16.61 \%)\end{array}$ & $\begin{array}{l}38,403 \\
(18.58 \%)\end{array}$ & $<0.001$ & $\begin{array}{l}17,997 \\
(10.53 \%)\end{array}$ & $\begin{array}{l}24,519 \\
(14.34 \%)\end{array}$ & $\begin{array}{l}28,264 \\
(16.53 \%)\end{array}$ & $\begin{array}{l}31,687 \\
(18.53 \%)\end{array}$ & $<0.001$ \\
\hline $\begin{array}{l}\text { Regional } \\
\text { Hospital }\end{array}$ & $\begin{array}{l}134,006 \\
(12.73 \%)\end{array}$ & $\begin{array}{l}71,877 \\
(16.95 \%)\end{array}$ & $\begin{array}{l}35,982 \\
(19.55 \%)\end{array}$ & $\begin{array}{l}48,697 \\
(23.56 \%)\end{array}$ & & $\begin{array}{l}22,169 \\
(12.97 \%)\end{array}$ & $\begin{array}{l}29,101 \\
(17.02 \%)\end{array}$ & $\begin{array}{l}33,523 \\
(19.61 \%)\end{array}$ & $\begin{array}{l}40,144 \\
(23.48 \%)\end{array}$ & \\
\hline Local Hospital & $\begin{array}{l}95,131 \\
(9.03 \%)\end{array}$ & $\begin{array}{l}47,445 \\
(11.19 \%)\end{array}$ & $\begin{array}{l}22,546 \\
(12.25 \%)\end{array}$ & $\begin{array}{l}29,415 \\
(14.23 \%)\end{array}$ & & $\begin{array}{l}15,974 \\
(9.34 \%)\end{array}$ & $\begin{array}{l}19,405 \\
(11.35 \%)\end{array}$ & $\begin{array}{l}21,014 \\
(12.29 \%)\end{array}$ & $\begin{array}{l}24,152 \\
(14.13 \%)\end{array}$ & \\
\hline Clinics & $\begin{array}{l}718,568 \\
(68.24 \%)\end{array}$ & $\begin{array}{l}244,632 \\
(57.69 \%)\end{array}$ & $\begin{array}{l}94,921 \\
(51.58 \%)\end{array}$ & $\begin{array}{l}90,183 \\
(43.63 \%)\end{array}$ & & $\begin{array}{l}114,839 \\
(67.17 \%)\end{array}$ & $\begin{array}{l}97,954 \\
(57.29 \%)\end{array}$ & $\begin{array}{l}88,178 \\
(51.57 \%)\end{array}$ & $\begin{array}{l}74,996 \\
(43.86 \%)\end{array}$ & \\
\hline
\end{tabular}

Mammography

certification $(\mathrm{N}$,

$\%)$

\begin{tabular}{|c|c|c|c|c|c|c|c|c|c|c|}
\hline No & $\begin{array}{l}780,993 \\
(74.17 \%)\end{array}$ & $\begin{array}{l}277,076 \\
(65.34 \%)\end{array}$ & $\begin{array}{l}110,768 \\
(60.20 \%)\end{array}$ & $\begin{array}{l}111,189 \\
(53.79 \%)\end{array}$ & $<0.001$ & $\begin{array}{l}125,451 \\
(73.37 \%)\end{array}$ & $\begin{array}{l}111,359 \\
(65.13 \%)\end{array}$ & $\begin{array}{l}103,045 \\
(60.27 \%)\end{array}$ & $\begin{array}{l}92,184 \\
(53.92 \%)\end{array}$ & $<0.001$ \\
\hline Yes & $\begin{array}{l}272,025 \\
(25.83 \%)\end{array}$ & $\begin{array}{l}146,996 \\
(34.66 \%)\end{array}$ & $\begin{array}{l}73,246 \\
(39.80 \%)\end{array}$ & $\begin{array}{l}95,509 \\
(46.21 \%)\end{array}$ & & $\begin{array}{l}45,528 \\
(26.63 \%)\end{array}$ & $\begin{array}{l}59,620 \\
(34.87 \%)\end{array}$ & $\begin{array}{l}67,934 \\
(39.73 \%)\end{array}$ & $\begin{array}{l}78,795 \\
(46.08 \%)\end{array}$ & \\
\hline
\end{tabular}

$\mathrm{NHI}$ branch (N,

$\%)$

\begin{tabular}{|c|c|c|c|c|c|c|c|c|c|c|}
\hline Taipei & $\begin{array}{l}371,797 \\
(35.31 \%)\end{array}$ & $\begin{array}{l}148,509 \\
(35.02 \%)\end{array}$ & $\begin{array}{l}62,989 \\
(34.23 \%)\end{array}$ & $\begin{array}{l}64,526 \\
(31.22 \%)\end{array}$ & $<0.001$ & $\begin{array}{l}59,305 \\
(34.69 \%)\end{array}$ & $\begin{array}{l}59,623 \\
(34.87 \%)\end{array}$ & $\begin{array}{l}58,171 \\
(34.02 \%)\end{array}$ & $\begin{array}{l}53,419 \\
(31.24 \%)\end{array}$ & 1.000 \\
\hline Northern & $\begin{array}{l}146,004 \\
(13.87 \%)\end{array}$ & $\begin{array}{l}58,190 \\
(13.72 \%)\end{array}$ & $\begin{array}{l}24,402 \\
(13.26 \%)\end{array}$ & $\begin{array}{l}27,358 \\
(13.24 \%)\end{array}$ & & $\begin{array}{l}23,801 \\
(13.92 \%)\end{array}$ & $\begin{array}{l}23,336 \\
(13.65 \%)\end{array}$ & $\begin{array}{l}22,828 \\
(13.35 \%)\end{array}$ & $\begin{array}{l}22,401 \\
(13.10 \%)\end{array}$ & \\
\hline Central & $\begin{array}{l}191,240 \\
(18.16 \%)\end{array}$ & $\begin{array}{l}74,823 \\
(17.64 \%)\end{array}$ & $\begin{array}{l}32,048 \\
(17.42 \%)\end{array}$ & $\begin{array}{l}38,248 \\
(18.50 \%)\end{array}$ & & $\begin{array}{l}30,933 \\
(18.09 \%)\end{array}$ & $\begin{array}{l}30,583 \\
(17.89 \%)\end{array}$ & $\begin{array}{l}29,905 \\
(17.49 \%)\end{array}$ & $\begin{array}{l}31,599 \\
(18.48 \%)\end{array}$ & \\
\hline Southern & $\begin{array}{l}138,465 \\
(13.15 \%)\end{array}$ & $\begin{array}{l}60,606 \\
(14.29 \%)\end{array}$ & $\begin{array}{l}28,523 \\
(15.50 \%)\end{array}$ & $\begin{array}{l}32,241 \\
(15.60 \%)\end{array}$ & & $\begin{array}{l}22,928 \\
(13.41 \%)\end{array}$ & $\begin{array}{l}24,322 \\
(14.23 \%)\end{array}$ & $\begin{array}{l}26,469 \\
(15.48 \%)\end{array}$ & $\begin{array}{l}26,695 \\
(15.61 \%)\end{array}$ & \\
\hline Kao-Ping & $\begin{array}{l}184,951 \\
(17.56 \%)\end{array}$ & $\begin{array}{l}71,427 \\
(16.84 \%)\end{array}$ & $\begin{array}{l}30,499 \\
(16.57 \%)\end{array}$ & $\begin{array}{l}37,690 \\
(18.23 \%)\end{array}$ & & $\begin{array}{l}30,641 \\
(17.92 \%)\end{array}$ & $\begin{array}{l}28,985 \\
(16.95 \%)\end{array}$ & $\begin{array}{l}28,454 \\
(16.64 \%)\end{array}$ & $\begin{array}{l}31,432 \\
(18.38 \%)\end{array}$ & \\
\hline Eastern & $\begin{array}{l}20,561 \\
(1.95 \%)\end{array}$ & $\begin{array}{l}10,517 \\
(2.48 \%)\end{array}$ & $\begin{array}{l}5,553 \\
(3.02 \%)\end{array}$ & $\begin{array}{l}6,635 \\
(3.21 \%)\end{array}$ & & $\begin{array}{l}3,371 \\
(1.97 \%)\end{array}$ & $\begin{array}{l}4,130 \\
(2.42 \%)\end{array}$ & $\begin{array}{l}5,152 \\
(3.01 \%)\end{array}$ & $\begin{array}{l}5,433 \\
(3.18 \%)\end{array}$ & \\
\hline
\end{tabular}

Note: $\mathrm{CCl}=$ modified Charlson comorbidity index, $\mathrm{NHI}=$ National Health Insurance Administration, NTD=New Taiwanese Dollar.

a Exact matching approach was used to 1-to-1 match groups of samples with different level of overall chronic illness severity based on their index age in years and baseline income status.

${ }^{b}$ P-value for comparison of patients' and health care providers' characteristics between screened and non-screened women, chi-square tests were used for categorical variables and t-tests for continuous variables.

${ }^{\mathrm{c}}$ Income status was presented in New Taiwanese Dollar (NTD). The exchange rate between NTD and US Dollar is about 30:1 in this study. 
Table 2 compares mammography participation, newly diagnosed breast cancer stages, and all-cause mortality among women at different levels of chronical illness severity. The Cochran-Armitage test for trend was used to compare frequencies of outcome measures across chronic illness levels. Mammography rates and repeat participation rates decreased as chronic illness levels increased ( $p<0.001)$.

Approximately $0.92-1.25 \%$ of matched cohorts at each CCl level were newly diagnosed with breast cancer. The proportion of early breast cancer diagnosis decreased as the chronic illness level increased $(p<0.001)$, while the all-cause mortality rate increased $(p<0.001)$. 
Table 2

Associations between different levels of chronic illness and outcomes of interest among matched cohorts

\begin{tabular}{|c|c|c|c|c|c|}
\hline \multirow[b]{2}{*}{ Variables } & \multicolumn{5}{|c|}{ Matched Cohort } \\
\hline & $\mathrm{CCl}=0$ & $\mathrm{CCl}=1$ & $\mathrm{CCl}=2$ & $\mathrm{CCl}=3+$ & \multirow{2}{*}{$\begin{array}{l}\mathrm{p}- \\
\text { value }^{\mathrm{a}}\end{array}$} \\
\hline $\mathrm{N}$ & 170,979 & 170,979 & 170,979 & 170,979 & \\
\hline \multicolumn{6}{|l|}{ Mammography Utilization } \\
\hline \multicolumn{6}{|l|}{ Mammography participation during follow-up period (N, \%) } \\
\hline No & $\begin{array}{l}90,925 \\
(53.18 \%)\end{array}$ & $\begin{array}{l}99,169 \\
(58.00 \%)\end{array}$ & $\begin{array}{l}107,156 \\
(62.67 \%)\end{array}$ & $\begin{array}{l}125,604 \\
(73.46 \%)\end{array}$ & $<0.001$ \\
\hline Yes & $\begin{array}{l}80,054 \\
(46.82 \%)\end{array}$ & $\begin{array}{l}71,810 \\
(42.00 \%)\end{array}$ & $\begin{array}{l}63,823 \\
(37.33 \%)\end{array}$ & $\begin{array}{l}45,375 \\
(26.54 \%)\end{array}$ & \\
\hline \multicolumn{6}{|l|}{ Repeated Mammography during follow-up period (N, \%) } \\
\hline No repeated mammography & $\begin{array}{l}138,191 \\
(80.82 \%)\end{array}$ & $\begin{array}{l}145,830 \\
(85.29 \%)\end{array}$ & $\begin{array}{l}151,285 \\
(88.48 \%)\end{array}$ & $\begin{array}{l}159,979 \\
(93.57 \%)\end{array}$ & $<0.001$ \\
\hline $\begin{array}{l}\text { Repeated mammography (at least twice during the observation } \\
\text { period) }\end{array}$ & $\begin{array}{l}32,788 \\
(19.18 \%)\end{array}$ & $\begin{array}{l}25,149 \\
(14.71 \%)\end{array}$ & $\begin{array}{l}19,694 \\
(11.52 \%)\end{array}$ & $\begin{array}{l}11,000 \\
(6.43 \%)\end{array}$ & \\
\hline \multicolumn{6}{|l|}{$\begin{array}{l}\text { Repeated Mammography during follow-up period among those } \\
\text { received mammography }(\mathrm{N}, \%)\end{array}$} \\
\hline No repeated mammography & $\begin{array}{l}47,266 \\
(59.04 \%)\end{array}$ & $\begin{array}{l}46,661 \\
(64.98 \%)\end{array}$ & $\begin{array}{l}44,129 \\
(69.14 \%)\end{array}$ & $\begin{array}{l}34,375 \\
(75.76 \%)\end{array}$ & $<0.001$ \\
\hline $\begin{array}{l}\text { Repeated mammography (at least twice during the observation } \\
\text { period) }\end{array}$ & $\begin{array}{l}32,788 \\
(40.96 \%)\end{array}$ & $\begin{array}{l}25,149 \\
(35.02 \%)\end{array}$ & $\begin{array}{l}19,694 \\
(30.86 \%)\end{array}$ & $\begin{array}{l}11,000 \\
(24.24 \%)\end{array}$ & \\
\hline \multicolumn{6}{|l|}{$\begin{array}{l}\text { Assess of mammography services among those received } \\
\text { mammography }(\mathrm{N}, \%)\end{array}$} \\
\hline Inreach (through in-hospital examination) & $\begin{array}{l}43,739 \\
(54.64 \%)\end{array}$ & $\begin{array}{l}41,720 \\
(58.10 \%)\end{array}$ & $\begin{array}{l}38,861 \\
(60.89 \%)\end{array}$ & $\begin{array}{l}28,355 \\
(62.49 \%)\end{array}$ & $<0.001$ \\
\hline Outreach (through mammography car) & $\begin{array}{l}36,315 \\
(45.36 \%)\end{array}$ & $\begin{array}{l}30,090 \\
(41.90 \%)\end{array}$ & $\begin{array}{l}24,962 \\
(39.11 \%)\end{array}$ & $\begin{array}{l}17,020 \\
(37.51 \%)\end{array}$ & \\
\hline \multicolumn{6}{|l|}{ Breast Cancer Diagnoses } \\
\hline \multicolumn{6}{|l|}{ Newly diagnosed as breast cancer (N, \%) } \\
\hline No & $\begin{array}{l}168,834 \\
(98.75 \%)\end{array}$ & $\begin{array}{l}169,301 \\
(99.02 \%)\end{array}$ & $\begin{array}{l}169,211 \\
(98.97 \%)\end{array}$ & $\begin{array}{l}169,404 \\
(99.08 \%)\end{array}$ & $<0.001$ \\
\hline Yes & $\begin{array}{l}2,145 \\
(1.25 \%)\end{array}$ & $\begin{array}{l}1,678 \\
(0.98 \%)\end{array}$ & $\begin{array}{l}1,768 \\
(1.03 \%)\end{array}$ & $\begin{array}{l}1,575 \\
(0.92 \%)\end{array}$ & \\
\hline \multicolumn{6}{|l|}{ Breast cancer stage $(\mathrm{N}, \%)^{\mathrm{c}}$} \\
\hline Stage 0 & $\begin{array}{l}284 \\
(13.24 \%)\end{array}$ & $\begin{array}{l}267 \\
(15.91 \%)\end{array}$ & $\begin{array}{l}235 \\
(13.29 \%)\end{array}$ & $\begin{array}{l}152 \\
(9.65 \%)\end{array}$ & $<0.001$ \\
\hline Stage I & $\begin{array}{l}650 \\
(30.30 \%)\end{array}$ & $\begin{array}{l}562 \\
(33.49 \%)\end{array}$ & $\begin{array}{l}511 \\
(28.90 \%)\end{array}$ & $\begin{array}{l}394 \\
(25.02 \%)\end{array}$ & \\
\hline Stage II & $\begin{array}{l}688 \\
(32.07 \%)\end{array}$ & $\begin{array}{l}514 \\
(30.63 \%)\end{array}$ & $\begin{array}{l}569 \\
(32.18 \%)\end{array}$ & $\begin{array}{l}432 \\
(27.43 \%)\end{array}$ & \\
\hline
\end{tabular}

Note: $\mathrm{CCl}=$ modified Charlson comorbidity index.

a P-value was generated by using Cochran-Armitage tests for trend.

b Misclassification were those TNM classifications in the national cancer registry, which were not missing, but with codes like "999/99" (unclear or physicians did not code), "888/88" (non-applicable), "BBB/BB/B" or "X" (TX, NX, MX, occult carcinoma, found cancer cell but may not a specific tumor).

c Only those with newly diagnosed as breast cancer were analyzed. 


\begin{tabular}{|c|c|c|c|c|c|}
\hline \multirow[b]{2}{*}{ Stage III } & \multicolumn{5}{|c|}{ Matched Cohort } \\
\hline & $\begin{array}{l}284 \\
(13.24 \%)\end{array}$ & $\begin{array}{l}188 \\
(11.20 \%)\end{array}$ & $\begin{array}{l}251 \\
(14.20 \%)\end{array}$ & $\begin{array}{l}227 \\
(14.41 \%)\end{array}$ & \\
\hline Stage IV & $\begin{array}{l}136 \\
(6.34 \%)\end{array}$ & 80 (4.77\%) & $\begin{array}{l}110 \\
(6.22 \%)\end{array}$ & $\begin{array}{l}278 \\
(17.65 \%)\end{array}$ & \\
\hline Missing values/ or misclassification ${ }^{b}$ & $\begin{array}{l}103 \\
(4.80 \%)\end{array}$ & $67(3.99 \%)$ & $92(5.20 \%)$ & $92(5.84 \%)$ & \\
\hline $\begin{array}{l}\text { Breast cancer early/late stage } \\
\text { (exclude missing) during follow-up peri }\end{array}$ & \multicolumn{4}{|c|}{ (exclude missing) during follow-up period $(\mathrm{N}, \%)^{\mathrm{c}}$} & \\
\hline Early Stage $(0,1,2)$ & $\begin{array}{l}1,622 \\
(79.43 \%)\end{array}$ & $\begin{array}{l}1,343 \\
(83.36 \%)\end{array}$ & $\begin{array}{l}1,315 \\
(78.46 \%)\end{array}$ & $\begin{array}{l}978 \\
(65.95 \%)\end{array}$ & $<0.001$ \\
\hline Late Stage $(3,4)$ & $\begin{array}{l}420 \\
(20.57 \%)\end{array}$ & $\begin{array}{l}268 \\
(16.64 \%)\end{array}$ & $\begin{array}{l}361 \\
(21.54 \%)\end{array}$ & $\begin{array}{l}505 \\
(34.05 \%)\end{array}$ & \\
\hline \multicolumn{6}{|l|}{ All-cause mortality (N, \%) } \\
\hline No & $\begin{array}{l}168,979 \\
(98.83 \%)\end{array}$ & $\begin{array}{l}168,028 \\
(98.27 \%)\end{array}$ & $\begin{array}{l}165,805 \\
(96.97 \%)\end{array}$ & $\begin{array}{l}155,997 \\
(91.24 \%)\end{array}$ & $<0.001$ \\
\hline Yes & $\begin{array}{l}2,000 \\
(1.17 \%)\end{array}$ & $\begin{array}{l}2,951 \\
(1.73 \%)\end{array}$ & $\begin{array}{l}5,174 \\
(3.03 \%)\end{array}$ & $\begin{array}{l}14,982 \\
(8.76 \%)\end{array}$ & \\
\hline \multicolumn{6}{|c|}{ Note: $\mathrm{CCl}=$ modified Charlson comorbidity index. } \\
\hline \multicolumn{6}{|c|}{ a P-value was generated by using Cochran-Armitage tests for trend. } \\
\hline \multicolumn{6}{|c|}{$\begin{array}{l}\text { b Misclassification were those TNM classifications in the national cancer registry, which were not missing, but with codes like " } 999 / 99 \text { " } \\
\text { (unclear or physicians did not code), "888/88" (non-applicable), "BBB/BB/B" or "X" (TX, NX, MX, occult carcinoma, found cancer cell but } \\
\text { may not a specific tumor). }\end{array}$} \\
\hline
\end{tabular}

Table 3 provides results from five conditional logistic regression models, which examined the effects of chronic illness on mammography participation (Model 1); and early diagnosis of breast cancer (Model 2); the interaction of $\mathrm{CCl}$ and mammography on early diagnosis of breast cancer (Model 3); all-cause mortality (Model 4); and the interaction effect on all-cause mortality (Model 5). Compared with CCI score 0 , women with more severe chronic conditions were less likely to participate in mammography screening, less likely to be diagnosed at an early stage of breast cancer, and at higher risk of all-cause mortality. Mammography participation increased the likelihood of early breast cancer diagnosis $(\mathrm{OR}=1.48,95 \% \mathrm{Cl}=1.36-1.60)$ and decreased risk of all-cause mortality $(\mathrm{OR}=0.63,95 \% \mathrm{Cl}=0.60-0.66)$. The interaction terms of $\mathrm{CCl}$ and mammography participation indicated statistically significantly increased benefits of early breast cancer diagnosis and decreased risk of all-cause mortality as chronic illness increased. 
Table 3

Conditional logistic regression model results for the effect of mammography uptake on early diagnosis of breast cancer and mortality prevention among women with different levels of chronic illness

\begin{tabular}{|c|c|c|c|c|c|c|c|c|c|c|}
\hline \multirow[t]{2}{*}{ Covariates/ Models } & \multicolumn{2}{|c|}{$\begin{array}{l}\text { Model 1: } \\
\text { Mammography } \\
\text { participation }\end{array}$} & \multicolumn{2}{|c|}{$\begin{array}{l}\text { Model 2: Early } \\
\text { diagnosis of } \\
\text { breast cancer }\end{array}$} & \multicolumn{2}{|c|}{$\begin{array}{l}\text { Model 3: Early } \\
\text { detection of breast } \\
\text { cancer (Interaction) }\end{array}$} & \multicolumn{2}{|c|}{$\begin{array}{l}\text { Model 4: All- } \\
\text { cause mortality }\end{array}$} & \multicolumn{2}{|c|}{$\begin{array}{l}\text { Model 5: All- } \\
\text { cause mortality } \\
\text { (Interaction) }\end{array}$} \\
\hline & $\begin{array}{l}\text { OR } \\
(95 \% \mathrm{Cl})\end{array}$ & $\begin{array}{l}\mathrm{p}- \\
\text { value }\end{array}$ & $\begin{array}{l}\text { OR } \\
(95 \% \mathrm{Cl})\end{array}$ & $\begin{array}{l}\mathrm{p}- \\
\text { value }\end{array}$ & $\begin{array}{l}\text { OR } \\
(95 \% \mathrm{Cl})\end{array}$ & $\begin{array}{l}\mathrm{p}- \\
\text { value }\end{array}$ & $\begin{array}{l}\text { OR } \\
(95 \% \mathrm{Cl})\end{array}$ & $\begin{array}{l}\mathrm{p}- \\
\text { value }\end{array}$ & $\begin{array}{l}\text { OR } \\
(95 \% \mathrm{Cl})\end{array}$ & $\begin{array}{l}\mathrm{p}- \\
\text { value }\end{array}$ \\
\hline \multicolumn{11}{|l|}{$\begin{array}{l}\text { Mammography } \\
\text { participation (ref.= No) }\end{array}$} \\
\hline Yes & & & $\begin{array}{l}1.48 \\
(1.36 \\
1.60)\end{array}$ & $<.0001$ & $\begin{array}{l}1.03 \\
(0.90,1.18)\end{array}$ & 0.672 & $\begin{array}{l}0.63 \\
(0.60 \\
0.66)\end{array}$ & $<.0001$ & $\begin{array}{l}0.95 \\
(0.86 \\
1.06)\end{array}$ & 0.353 \\
\hline \multicolumn{11}{|l|}{$\begin{array}{l}\text { Women' chronic illness } \\
\text { characteristics }\end{array}$} \\
\hline \multicolumn{11}{|l|}{$\begin{array}{l}\mathrm{CCl} \text { categories (Ref.: } \\
\mathrm{CCl}=0 \text { ) }\end{array}$} \\
\hline 1 & $\begin{array}{l}0.66 \\
(0.65 \\
0.67)\end{array}$ & $<0.001$ & $\begin{array}{l}0.80 \\
(0.74 \\
0.87)\end{array}$ & $<0.001$ & $\begin{array}{l}0.61(0.53 \\
0.69)\end{array}$ & $<0.001$ & $\begin{array}{l}1.44 \\
(1.35 \\
1.53)\end{array}$ & $<0.001$ & $\begin{array}{l}1.57 \\
(1.46 \\
1.69)\end{array}$ & $<0.001$ \\
\hline 2 & $\begin{array}{l}0.48 \\
(0.47 \\
0.49)\end{array}$ & $<0.001$ & $\begin{array}{l}0.79 \\
(0.73 \\
0.85)\end{array}$ & $<0.001$ & $\begin{array}{l}0.66(0.58 \\
0.75)\end{array}$ & $<0.001$ & $\begin{array}{l}2.40 \\
(2.27 \\
2.53)\end{array}$ & $<0.001$ & $\begin{array}{l}2.72 \\
(2.54 \\
2.91)\end{array}$ & $<0.001$ \\
\hline $3+$ & $\begin{array}{l}0.29 \\
(0.29 \\
0.30)\end{array}$ & $<0.001$ & $\begin{array}{l}0.60 \\
(0.55 \\
0.65)\end{array}$ & $<0.001$ & $\begin{array}{l}0.41(0.36 \\
0.47)\end{array}$ & $<0.001$ & $\begin{array}{l}6.38 \\
(6.07 \\
6.72)\end{array}$ & $<0.001$ & $\begin{array}{l}7.71 \\
(7.24 \\
8.22)\end{array}$ & $<0.001$ \\
\hline \multicolumn{11}{|l|}{$\begin{array}{l}\text { Interaction of } \\
\text { mammography } \\
\text { participation and levels } \\
\text { of chronic illness } \\
\text { burden }\end{array}$} \\
\hline $\begin{array}{l}\mathrm{CCl}: 1 \text { * mammography } \\
\text { participation }\end{array}$ & & & & & $\begin{array}{l}1.62(1.34, \\
1.96)\end{array}$ & $<.0001$ & & & $\begin{array}{l}0.78 \\
(0.68 \\
0.89)\end{array}$ & 0.0002 \\
\hline $\begin{array}{l}\mathrm{CCl}: 2 \text { * mammography } \\
\text { participation }\end{array}$ & & & & & $\begin{array}{l}1.35(1.12, \\
1.62)\end{array}$ & 0.002 & & & $\begin{array}{l}0.68 \\
(0.60 \\
0.77)\end{array}$ & $<0.001$ \\
\hline $\begin{array}{l}\text { CCl: } 3+* \\
\text { mammography } \\
\text { participation }\end{array}$ & & & & & $\begin{array}{l}2.08(1.70 \\
2.54)\end{array}$ & $<0.001$ & & & $\begin{array}{l}0.47 \\
(0.41 \\
0.53)\end{array}$ & $<0.001$ \\
\hline \multicolumn{11}{|l|}{$\begin{array}{l}\text { Women' health } \\
\text { behavioral } \\
\text { characteristics }\end{array}$} \\
\hline \multicolumn{11}{|l|}{$\begin{array}{l}\text { Receiving population- } \\
\text { based pap smear } \\
\text { screening within follow } \\
\text { up period (Ref.: No) }\end{array}$} \\
\hline Yes & $\begin{array}{l}11.05 \\
(10.87 \\
11.25)\end{array}$ & $<0.001$ & $\begin{array}{l}2.22 \\
(2.04 \\
2.41)\end{array}$ & $<0.001$ & $\begin{array}{l}2.24(2.06 \\
2.44)\end{array}$ & $<0.001$ & $\begin{array}{l}0.62 \\
(0.59 \\
0.64)\end{array}$ & $<0.001$ & $\begin{array}{l}0.61 \\
(0.59 \\
0.64)\end{array}$ & $<0.001$ \\
\hline $\begin{array}{l}\text { Receiving population- } \\
\text { based adult physical } \\
\text { examinations within } \\
\text { follow up period (Ref.: } \\
\text { No) }\end{array}$ & & & & & & & & & & \\
\hline
\end{tabular}

Note: Ref.=Reference group; $\mathrm{OR}=$ odds ratio; $\mathrm{Cl}=$ confidence interval. $\mathrm{NHI}=$ National Health Insurance $\mathrm{Administration}$; $\mathrm{CCl}=$ modified Charlson comorbidity index. 


\begin{tabular}{|c|c|c|c|c|c|c|c|c|c|c|}
\hline \multirow{2}{*}{$\begin{array}{l}\text { Covariates/ Models } \\
\text { Yes }\end{array}$} & \multicolumn{2}{|c|}{$\begin{array}{l}\text { Model 1: } \\
\text { Mammography } \\
\text { participation }\end{array}$} & \multicolumn{2}{|c|}{$\begin{array}{l}\text { Model 2: Early } \\
\text { diagnosis of } \\
\text { breast cancer }\end{array}$} & \multicolumn{2}{|c|}{$\begin{array}{l}\text { Model 3: Early } \\
\text { detection of breast } \\
\text { cancer (Interaction) }\end{array}$} & \multicolumn{2}{|c|}{$\begin{array}{l}\text { Model 4: All- } \\
\text { cause mortality }\end{array}$} & \multicolumn{2}{|c|}{$\begin{array}{l}\text { Model 5: All- } \\
\text { cause mortality } \\
\text { (Interaction) }\end{array}$} \\
\hline & $\begin{array}{l}1.80 \\
(1.78 \\
1.83)\end{array}$ & $<0.001$ & $\begin{array}{l}0.88 \\
(0.82 \\
0.95)\end{array}$ & 0.001 & $\begin{array}{l}0.89(0.83 \\
0.96)\end{array}$ & 0.003 & $\begin{array}{l}0.47 \\
(0.45 \\
0.49)\end{array}$ & $<0.001$ & $\begin{array}{l}0.46 \\
(0.44 \\
0.48)\end{array}$ & $<0.001$ \\
\hline \multicolumn{11}{|l|}{$\begin{array}{l}\text { Primary health care } \\
\text { providers' } \\
\text { characteristics }\end{array}$} \\
\hline \multicolumn{11}{|l|}{$\begin{array}{l}\text { Accreditation level } \\
\text { (Ref.: Medical Center) }\end{array}$} \\
\hline Regional Hospital & $\begin{array}{l}1.10 \\
(1.08 \\
1.13)\end{array}$ & $<0.001$ & $\begin{array}{l}0.72 \\
(0.66 \\
0.80)\end{array}$ & $<0.001$ & $\begin{array}{l}0.72(0.65 \\
0.79)\end{array}$ & $<0.001$ & $\begin{array}{l}0.83 \\
(0.78 \\
0.87)\end{array}$ & $<0.001$ & $\begin{array}{l}0.83 \\
(0.78 \\
0.88)\end{array}$ & $<0.001$ \\
\hline Local Hospital & $\begin{array}{l}1.04 \\
(1.01 \\
1.08)\end{array}$ & 0.011 & $\begin{array}{l}0.40 \\
(0.35 \\
0.47)\end{array}$ & $<0.001$ & $\begin{array}{l}0.40(0.35 \\
0.46)\end{array}$ & $<0.001$ & $\begin{array}{l}0.63 \\
(0.59 \\
0.68)\end{array}$ & $<0.001$ & $\begin{array}{l}0.63 \\
(0.59 \\
0.68)\end{array}$ & $<0.001$ \\
\hline Clinics & $\begin{array}{l}0.86 \\
(0.83 \\
0.89)\end{array}$ & $<0.001$ & $\begin{array}{l}0.32 \\
(0.27 \\
0.38)\end{array}$ & $<0.001$ & $\begin{array}{l}0.32(0.27 \\
0.38)\end{array}$ & $<0.001$ & $\begin{array}{l}0.38 \\
(0.35 \\
0.42)\end{array}$ & $<0.001$ & $\begin{array}{l}0.39 \\
(0.36 \\
0.42)\end{array}$ & $<0.001$ \\
\hline \multicolumn{11}{|l|}{$\begin{array}{l}\text { Mammography } \\
\text { certification (Ref.: No) }\end{array}$} \\
\hline Yes & $\begin{array}{l}1.59 \\
(1.54 \\
1.64)\end{array}$ & $<0.001$ & $\begin{array}{l}1.44 \\
(1.25 \\
1.66)\end{array}$ & $<0.001$ & $\begin{array}{l}1.44(1.25 \\
1.66)\end{array}$ & $<0.001$ & $\begin{array}{l}0.95 \\
(0.88 \\
1.01)\end{array}$ & 0.1066 & $\begin{array}{l}0.94 \\
(0.88 \\
1.01)\end{array}$ & 0.096 \\
\hline \multicolumn{11}{|l|}{$\begin{array}{l}\text { NHI branch (Ref.: } \\
\text { Taipei) }\end{array}$} \\
\hline Northern & $\begin{array}{l}1.12 \\
(1.09 \\
1.15)\end{array}$ & $<0.001$ & $\begin{array}{l}0.89 \\
(0.79 \\
0.99)\end{array}$ & 0.040 & $\begin{array}{l}0.89 \\
(0.79,1.00)\end{array}$ & 0.043 & $\begin{array}{l}1.44 \\
(1.36 \\
1.53)\end{array}$ & $<0.001$ & $\begin{array}{l}1.44 \\
(1.36 \\
1.53)\end{array}$ & $<0.001$ \\
\hline Central & $\begin{array}{l}0.99 \\
(0.97 \\
1.02)\end{array}$ & 0.613 & $\begin{array}{l}0.94 \\
(0.85 \\
1.04)\end{array}$ & 0.214 & $\begin{array}{l}0.94 \\
(0.85,1.04)\end{array}$ & 0.243 & $\begin{array}{l}1.50 \\
(1.42 \\
1.58)\end{array}$ & $<0.001$ & $\begin{array}{l}1.49 \\
(1.42 \\
1.58)\end{array}$ & $<0.001$ \\
\hline Southern & $\begin{array}{l}1.17 \\
(1.14 \\
1.20)^{\prime}\end{array}$ & $<0.001$ & $\begin{array}{l}0.97 \\
(0.87 \\
1.09)\end{array}$ & 0.609 & $\begin{array}{l}0.97 \\
(0.87,1.09)\end{array}$ & 0.595 & $\begin{array}{l}1.59 \\
(1.50 \\
1.68)\end{array}$ & $<0.001$ & $\begin{array}{l}1.59 \\
(1.50 \\
1.69)\end{array}$ & $<0.001$ \\
\hline Kao-Ping & $\begin{array}{l}1.09 \\
(1.07 \\
1.11)\end{array}$ & $<0.001$ & $\begin{array}{l}0.98 \\
(0.88 \\
1.08)\end{array}$ & 0.678 & $\begin{array}{l}0.98 \\
(0.88,1.08)\end{array}$ & 0.676 & $\begin{array}{l}1.63 \\
(1.54 \\
1.71)\end{array}$ & $<0.001$ & $\begin{array}{l}1.62 \\
(1.54 \\
1.71)\end{array}$ & $<0.001$ \\
\hline Eastern & $\begin{array}{l}1.17 \\
(1.12 \\
1.22)\end{array}$ & $<0.001$ & $\begin{array}{l}0.88 \\
(0.70 \\
1.10)\end{array}$ & 0.250 & $\begin{array}{l}0.88 \\
(0.70,1.10)\end{array}$ & 0.267 & $\begin{array}{l}1.97 \\
(1.77 \\
2.19)\end{array}$ & $<0.001$ & $\begin{array}{l}1.98 \\
(1.77 \\
2.20)\end{array}$ & $<0.001$ \\
\hline
\end{tabular}

Table 4 shows results of mean APPs for the probability of early breast cancer diagnosis and all-cause mortality among different chronic illness levels, and MEs of predicted probability between women who did and did not undergo mammography. The APPs of early diagnosis decreased for $\mathrm{CCl}$ scores $0,1,2,3+$ among both women with and without participating in mammography screening. Positive MEs of early breast cancer diagnosis between women who did and did not participate indicated the magnitude of benefit of mammography across chronic illness levels. The APPs of all-cause mortality increased for $\mathrm{CCl}$ scores $0,1,2,3+$ among women with and without participating in mammography screening. The MEs of all-cause mortality between women who did and did not participate at each chronic severity level indicated that mammography reduced the risk of mortality as chronic illness severity levels increased. 
Table 4

Results of adjusted predicted probabilities and marginal effects of the levels of chronic illness and probabilities of outcome of interests among matched cohorts with and without mammography participation

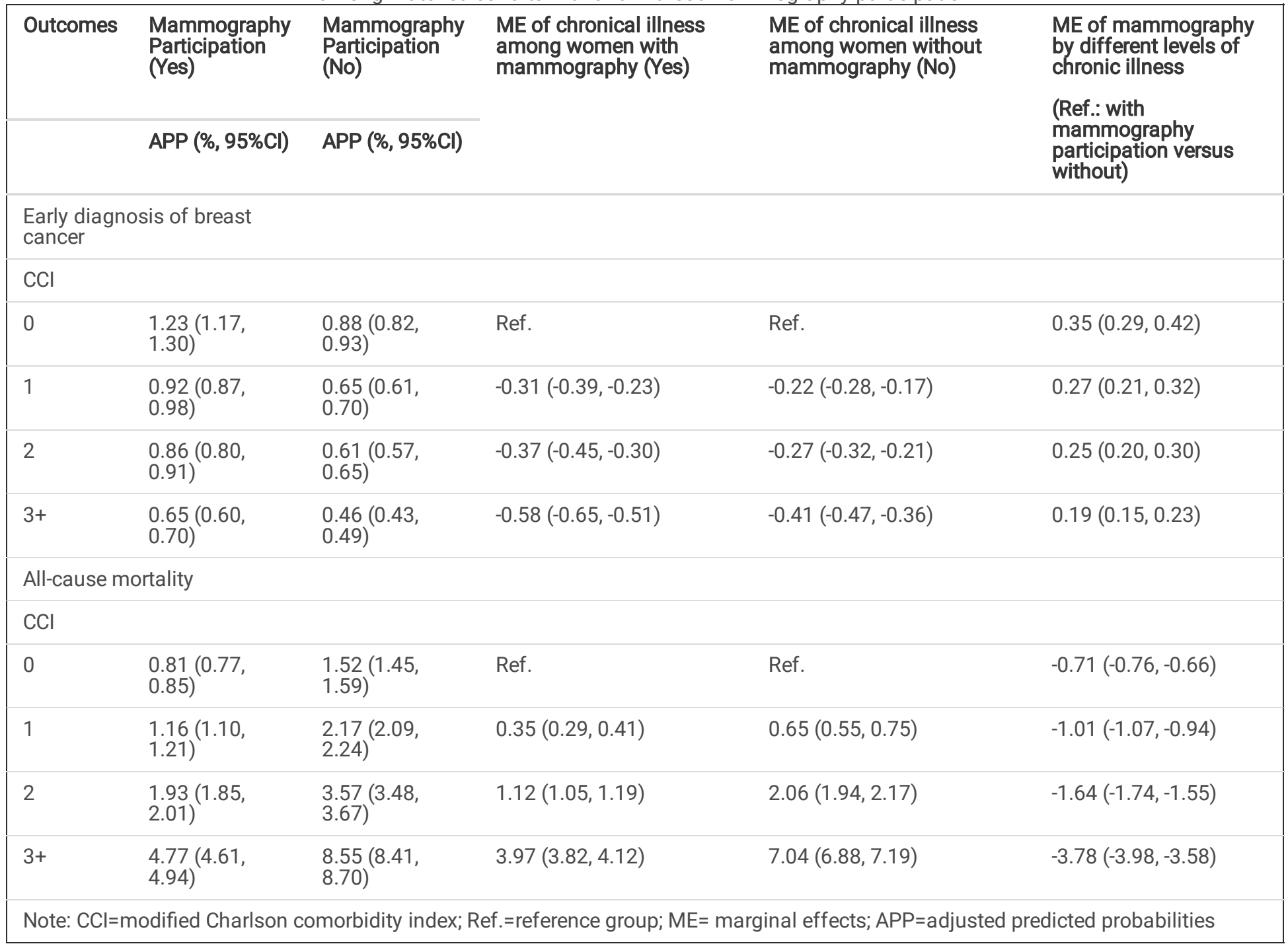

\section{Discussion}

This study examined potential benefits and moderation effects of mammography screening on early breast cancer diagnosis and mortality among women aged 50-69 years at various health statuses. A generic $\mathrm{CCl}$ measure was used to identify women at different levels of overall chronic illness burden. Consistent with previous literature, our findings indicate that women at higher chronic illness levels were less likely to participate in mammography screening and to have breast cancer newly diagnosed at early stages, and were at greater risk of allcause mortality. ${ }^{4,-9}$ Our findings further provide empirical evidence that mammography may moderate the association between chronic illness burden and early breast cancer diagnosis and mortality.

Comorbidity may present barriers to breast cancer screening and complicate diagnostic decision-making. ${ }^{7,10}$ Similar to findings from a systematic review and meta-analysis study based on few high-quality studies in Europe or in the United States by Diaz et al. (2017) and a recently study conducted in Taiwan by Hsieh (2021), ${ }^{10,11}$ our study findings indicate that women aged 50-69 years at higher chronic illness levels were less likely to participate in mammography screening in Taiwan. As Fleming et al. (2005) indicated, comorbidity as a predictor of newly diagnosed breast cancer stage may relate to several hypotheses: the interaction between comorbid conditions and cancers at the cellular level may increase risks of metastasis, or comorbid conditions may constitute a competing demand against use of preventive services. Additionally, as observed in clinical settings, physicians are more likely to request mammography for women at higher general risk 
of breast cancer (e.g., family history), but less likely to request it for average-risk women with chronic illnesses. ${ }^{7}$ These all may exacerbate the odds of late-stage breast cancer diagnosis and prognosis among women with chronic illness.

Existing studies suggest that mammography screening and reducing barriers to health system access may decrease the risks of late-stage diagnosis and mortality among women with chronic illness. Most of these existing studies focus on older women and were conducted in Europe and the United States. ${ }^{7,12,15,18,33}$ To the best of our knowledge, no studies specifically examined potential benefits of mammography screening for stage at diagnosis or mortality among younger women at different health statuses in Asian countries. In general, our findings confirm the benefit of mammography screening, which increased the likelihood of early stage breast cancer diagnosis by $48 \%$ and decreased odds of all-cause mortality by $37 \%$. Mammography screening significantly moderated the link between chronic illness burden and late-stage diagnosis and risk of all-cause mortality.

Our study has several strengths. It used four longitudinal nationwide population-based datasets linking NHI administrative claims, national cancer registry, death registry, and breast cancer screening registry in Taiwan. These included all women aged 50-69 years in 2010, or approximately 2.5 million population, and provided accurate screening attendance information for identifying screened and non-screened groups. In addition, the study database allowed us to generate a generic composite measure of total chronic illness burden from NHI administrative data, reducing potential recall bias from self-reported health status, ${ }^{10}$ or missing information due to using data from regional hospital-based electronic medical records. ${ }^{18}$ In addition, Czwikla et al. (2018) addressed methodology concern of selection bias issue as the results of mammography screening participants and nonparticipants are not comparable regarding various health statuses. ${ }^{33}$ To avoid potential selection bias as Czwikla et al. (2018) point out, we used an exact matching approach to generate balanced groups with different burdens of chronic conditions based on birth years and income status and compared outcomes of interest at the same chronic condition levels between screened and non-screened groups.

Nevertheless, this study also has several limitations. First, to compare results with existing studies, ${ }^{12,18}$ we used the CCI to measure overall burden of chronic conditions. Future studies may use other types of comorbidity measures to investigate benefits of mammography among women at different health statuses. Second, some unobservable confounders are unavailable when investigating research questions using secondary administrative data, such as lifestyle factors and habits or breast cancer awareness. Finally, the data were from women aged 50-69 years in Taiwan. Results may not generalize to other health systems in other countries.

In conclusion, analyzing national population-based data in Taiwan, this study provides empirical evidence with respect to the moderation effect of mammography screening, which increased likelihood of early stage breast cancer diagnosis and decreased odds of all-cause mortality. Public health policy and strategies may be necessary to improve mammography participation and early detection efforts for women with comorbid conditions.

\section{Declarations}

Funding Source: This study was supported by the Ministry of Science and Technology grant (M0ST105-2314-B-037-063, MOST106-2314-B037-036, MOST 108-2410-H-037 -007, MOST 109-2423-H-037-001-SS3). This study is supported partially by the Kaohsiung Medical University Research Foundation (KMU-M108007), the Kaohsiung Medical University Research Center Grant (KMU-KMU-TC109B08),the Research Center for Environmental Medicine from the Featured Area Research Center Program within the framework of the Higher Education Sprout Project by the Ministry of Education (MOE) in Taiwan, and Kaohsiung Medical University Research Center Grant (KMUTC109A01-1). I am also grateful to Kaohsiung Medical University for providing administrative and funding support. The author thanks the help from the Division of Medical Statistics and Bioinformatics, Department of Medical Research, Kaohsiung Medical University Hospital, and Center for Big Data Research (KMU-KMU-TC109B08), Kaohsiung Medical University for providing administrative support. None of which had any role in study design, data collection and analysis, decision to publish, or preparation of the manuscript.

Conflicts of interest/Ethical approval: We assure that each author meets authorship requirements. We declare that none of the authors has a conflict of interest with regard to this manuscript.

Financial Disclosure: The authors declare that they have no conflict of interest.

\section{(For Original Research Only) Specific contributions:}

Conception or design of the work, the acquisition, analysis: $\mathrm{HMH}$

Interpretation of data for the work: HMH, CTS, FMC

Page $14 / 17$ 
Revising it critically: HMH, CTS, FMC

Final approval: $\mathrm{HMH}, \mathrm{CTS}, \mathrm{FMC}$

Availability of Data and Materials: Not applicable

\section{References}

1. Sung, H. et al. Global Cancer Statistics 2020: GLOBOCAN Estimates of Incidence and Mortality Worldwide for 36 Cancers in 185 Countries. CA Cancer J Clin 71, 209-249, doi:10.3322/caac.21660 (2021).

2. HPA. 2017 Taiwan Cancer Registry Annual Report in Taiwan (Health Promotion Administration, Ministry of Health). Available at https://www.hpa.gov.tw/Pages/Detail.aspx?nodeid=269\&pid=13498 (Access on Sep 02, 2021) (2020).

3. Bhoo-Pathy, N. et al. Breast cancer research in Asia: adopt or adapt Western knowledge? European journal of cancer (Oxford, England: 1990) 49, 703-709, doi:10.1016/j.ejca.2012.09.014 (2013).

4. Sarfati, D., Koczwara, B. \& Jackson, C. The impact of comorbidity on cancer and its treatment. CA Cancer J Clin 66, 337-350, doi:10.3322/caac.21342 (2016).

5. Yancik, R., Ganz, P. A., Varricchio, C. G. \& Conley, B. Perspectives on comorbidity and cancer in older patients: approaches to expand the knowledge base. J Clin Onco/ 19, 1147-1151, doi:10.1200/JC0.2001.19.4.1147 (2001).

6. Yancik, R. et al. Effect of age and comorbidity in postmenopausal breast cancer patients aged 55 years and older. JAMA 285, 885-892, doi:10.1001/jama.285.7.885 (2001).

7. Fleming, S. T., Pursley, H. G., Newman, B., Pavlov, D. \& Chen, K. Comorbidity as a predictor of stage of illness for patients with breast cancer. Med Care 43, 132-140, doi:10.1097/00005650-200502000-00006 (2005).

8. Gurney, J., Sarfati, D. \& Stanley, J. The impact of patient comorbidity on cancer stage at diagnosis. Br J Cancer 113, 1375-1380, doi:10.1038/bjc.2015.355 (2015).

9. Terret, C., Castel-Kremer, E., Albrand, G. \& Droz, J. P. Effects of comorbidity on screening and early diagnosis of cancer in elderly people. The Lancet. Oncology 10, 80-87, doi:10.1016/S1470-2045(08)70336-X (2009).

10. Diaz, A. et al. Association between comorbidity and participation in breast and cervical cancer screening: A systematic review and meta-analysis. Cancer Epidemio/ 47, 7-19, doi:10.1016/j.canep.2016.12.010 (2017).

11. Hsieh, H. M. Effect of accessibility improvement in a national population-based breast cancer screening policy on mammography utilization among women with comorbid conditions in Taiwan. Soc Sci Med 284, 114245, doi:10.1016/j.socscimed.2021.114245 (2021).

12. Demb, J. et al. Screening Mammography Outcomes: Risk of Breast Cancer and Mortality by Comorbidity Score and Age. Journal of the National Cancer Institute 112, 599-606, doi:10.1093/jnci/djz172 (2020).

13. Duffy, S. et al. Annual mammographic screening to reduce breast cancer mortality in women from age 40 years: long-term follow-up of the UK Age RCT. Health technology assessment (Winchester, England) 24, 1-24, doi:10.3310/hta24550 (2020).

14. Duffy, S. W. et al. Effect of mammographic screening from age 40 years on breast cancer mortality (UK Age trial): final results of a randomised, controlled trial. The Lancet. Oncology 21, 1165-1172, doi:10.1016/S1470-2045(20)30398-3 (2020).

15. Braithwaite, D., Walter, L. C., Izano, M. \& Kerlikowske, K. Benefits and Harms of Screening Mammography by Comorbidity and Age: A Qualitative Synthesis of Observational Studies and Decision Analyses. J Gen Intern Med 31, 561-572, doi:10.1007/s11606-015-3580-3 (2016).

16. McCarthy, E. P. et al. Mammography use, breast cancer stage at diagnosis, and survival among older women. J Am Geriatr Soc 48, 1226-1233, doi:10.1111/j.1532-5415.2000.tb02595.x (2000).

17. Garcia-Albeniz, X. et al. Continuation of Annual Screening Mammography and Breast Cancer Mortality in Women Older Than 70 Years. Ann Intern Med 172, 381-389, doi:10.7326/M18-1199 (2020).

18. Beau, A. B. et al. Impact of chronic diseases on effect of breast cancer screening. Cancer Med 9, 3995-4003, doi:10.1002/cam4.3036 (2020).

19. Yen, A. M. et al. Population-Based Breast Cancer Screening With Risk-Based and Universal Mammography Screening Compared With Clinical Breast Examination: A Propensity Score Analysis of 1429890 Taiwanese Women. JAMA oncology 2, 915-921, doi:10.1001/jamaoncol.2016.0447 (2016).

20. Lee, Y. C. et al. The impact of universal National Health Insurance on population health: the experience of Taiwan. BMC health services research 10, 225, doi:10.1186/1472-6963-10-225 (2010).

Page 15/17 
21. Chiang, C. J. et al. Cancer trends in Taiwan. Jpn J Clin Oncol 40, 897-904, doi:10.1093/jjco/hyq057 (2010).

22. Chiang, C. J. et al. Incidence and survival of adult cancer patients in Taiwan, 2002-2012. Journal of the Formosan Medical Association = Taiwan yi zhi 115, 1076-1088, doi:10.1016/j.jfma.2015.10.011 (2016).

23. Deyo, R. A., Cherkin, D. C. \& Ciol, M. A. Adapting a clinical comorbidity index for use with ICD-9-CM administrative databases. J Clin Epidemiol 45, 613-619 (1992).

24. Quan, H. et al. Coding algorithms for defining comorbidities in ICD-9-CM and ICD-10 administrative data. Med Care 43, 1130-1139, doi:10.1097/01.mlr.0000182534.19832.83 (2005).

25. Stuart, E. A. Matching methods for causal inference: A review and a look forward. Stat Sci 25, 1-21, doi:10.1214/09-STS313 (2010).

26. HPA. Aging and Chronic Disease Control. Available at https://www.hpa.gov.tw/ (Access date: Jan 19, 2021) (2021).

27. Pham, H. H., Schrag, D., O'Malley, A. S., Wu, B. \& Bach, P. B. Care patterns in Medicare and their implications for pay for performance. N Engl J Med 356, 1130-1139, doi:10.1056/NEJMsa063979 (2007).

28. Armitage, P. Tests for Linear Trends in Proportions and Frequencies. Biometrics 11, 375-386 (1955).

29. Kuo, C. L., Duan, Y. \& Grady, J. Unconditional or Conditional Logistic Regression Model for Age-Matched Case-Control Data? Front Public Health 6, 57, doi:10.3389/fpubh.2018.00057 (2018).

30. Baron, R. M. \& Kenny, D. A. The Moderator-Mediator Variable Distrinction in Social Psychological Research: Conceptual, Strategic, and Statistical Considerations. Journal of Personality and Psychology 51, 1173-1182 (1986).

31. Norton, E. C., Dowd, B. E. \& Maciejewski, M. L. Marginal Effects-Quantifying the Effect of Changes in Risk Factors in Logistic Regression Models. JAMA 321, 1304-1305, doi:10.1001/jama.2019.1954 (2019).

32. Muller, C. J. \& MacLehose, R. F. Estimating predicted probabilities from logistic regression: different methods correspond to different target populations. Int J Epidemiol 43, 962-970, doi:10.1093/ije/dyu029 (2014).

33. Czwikla, J. et al. A cohort study of mammography screening finds that comorbidity measures are insufficient for controlling selection bias. J Clin Epidemio/ 104, 1-7, doi:10.1016/j.jclinepi.2018.07.014 (2018).

\section{Figures}


To identify distinct eligible women aged 50-69 years in the National

Health Insurance enrollment file during the patient identification

period in $2010(N=2,564,252)$

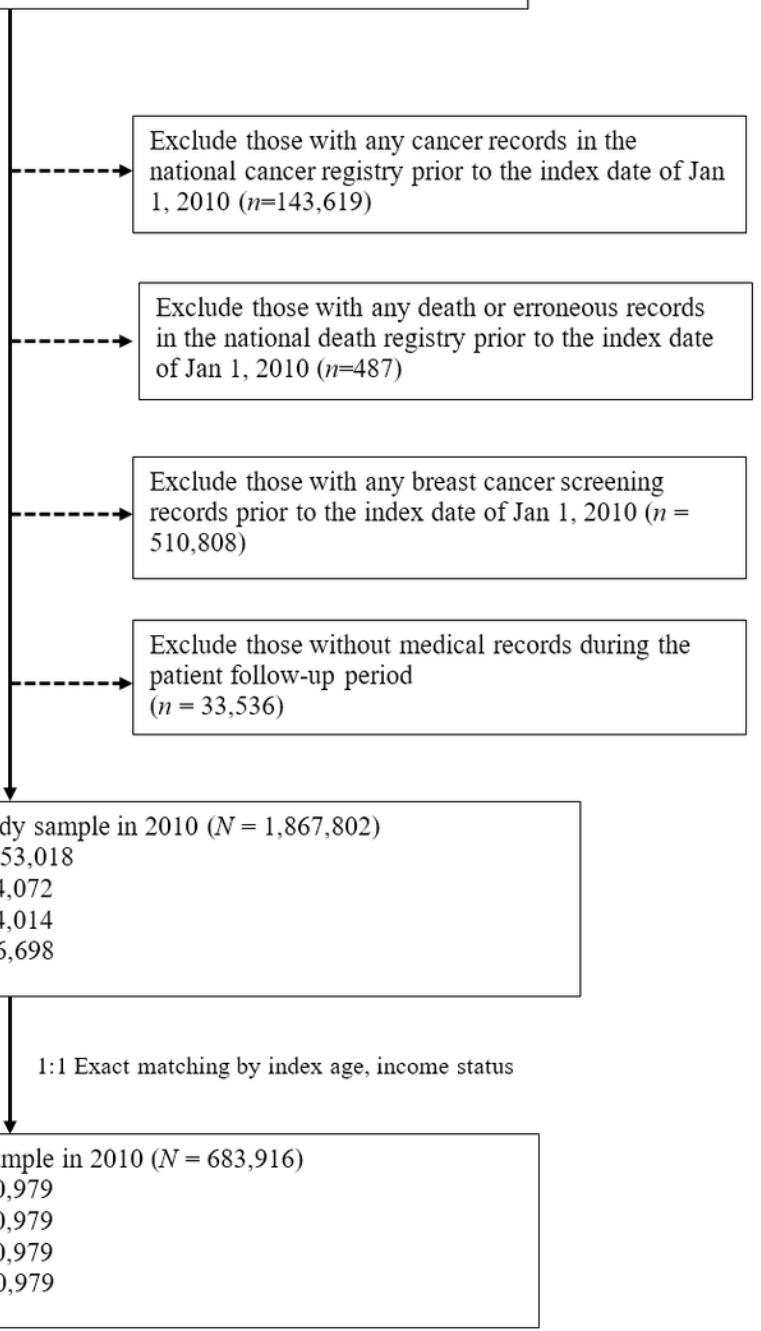

\section{Figure 1}

Inclusion and exclusion criteria in this study 